Part of Journal of Research of the National Bureau of Standards, Volume 33, September 1944

\title{
TRASNFORMATIONS OF THE FUNDAMENTAL EQUATIONS OF THERMODYNAMICS
}

\author{
By Floyd Buckley
}

\section{ABSTRACT}

A substitution group for generating families of thermodynamic formulas is derived. The method of derivation is based upon the transformation properties of a "group of functions" under a contact transformation. There exists a characteristic function and a "group of functions" for each representation, that is, each coordinate system, and to each function of the group there is an associated contact transformation which transforms the group into its equivalent in another representation. The invariance of the functional form of the characteristic groups of functions under contact transformations is equivalent to invariance under a substitution group $\mathscr{G} *$ on the space $(E H F G)(V-S-T P)$. The group $\mathscr{G} *$ is independent of the representation and can be generated geometrically.

There are four contact transformations (including the identity) associated with each representation. These transformations are equivalent, and from them families of equations can be found which are invariant under the group $\mathscr{G} *$. Other families can be found among the transformation formulae for the higher derivatives.

Formulas deducible by simple operations, for example, differentiation, on the characteristic group of functions of a given representation provide basic forms for families invariant under the group $\mathscr{G}^{*}$. The number of members in a family is $1,2,4$, or 8 .

\section{CONTENTS}

I. Introduction

II. The fundamental equations

III. The problem

IV. Tangential transformations

V. The Legendre transformations

VI. Thermodynamic formulas

VII. References_...

\section{INTRODUCTION}

The existence of symmetry relations between certain sets of thermodynamic formulas has been recognized since the classical work of Gibbs. Nevertheless, Koenig [1] ${ }^{1}$ seems to have been the first to exhibit these relations in the compact form of a substitution group on the thermodynamic functions $E, H, F, G$ and the variables of state $V, S$, $P, T ; N_{i}$. The method of derivation was not given, although it was shown how the group can be generated from the symmetry properties of a square. The purpose of this article is to present a derivation of Koenig's substitution group by making use of the transformation properties of the fundamental thermodynamic equations. The transformations employed are the Cylindrical Tangential (Contact) Transformations studied by Lie. 


\section{THE FUNDAMENTAL EQUATIONS}

The necessary assumptions are those given by Caratheodory [2] in his axiomatic development of the general theory. The following discussion does not require an analysis of these assumptions. Nor does it require a description of the methods used in establishing the relation between the general mathematical structure and experimental thermodynamics, particularly the determination of the characteristic function associated with a selected set of physical and chemical variables. It will suffice to recall that in a given description, that is, with a given choice of variables, there exists a characteristic function $Z$ appropriate to a given physical system, which is the solution of an exact differential equation of the Pfaff type:

$$
d Z-\sum_{i} X_{i} d X_{i}=0
$$

Once 7. has been found, the general behavior of the system can be conveniently described with the aid of auxiliary functions defined in terms of 7. and its partial derivatives. For example, in a reference system (description) using $V, S ; N_{i}$ as independent variables, all general thermodynamic formulas, that is, those formulas independent of an equation of state, are derivable from the characteristic Energy function, $E$, and the basic equations

$$
\begin{aligned}
& H \equiv E-V \frac{\partial E}{\partial V} \\
& F \equiv E-S \frac{\partial E}{\partial S} \\
& G \equiv E-V \frac{\partial E}{\partial V}-S \frac{\partial E}{\partial S}=H+F-E .
\end{aligned}
$$

The Energy function is a function only of the state of the system and satisfies the conditions:

$$
\begin{aligned}
& \text { Equilibrium State } \ldots \ldots d E=0 \quad \delta V=0 \quad \delta S=0 \quad \delta N_{i}=0 . \\
& \text { Reversible Process..... } \\
& \text { Natural Process } \\
& \text { Unnatural Process_...... } d E=0 \quad \delta V=0 \quad \delta S>0 \quad \delta N_{i}=0 .
\end{aligned}
$$

The functions $H, F, G$ are not uniquely defined, but in practical application the arbitrariness drops out. Legitimate operations on equations 1 generate a collection $\mathrm{C}$, of formulas valid in the description $V, S ; N_{i}$.

\section{THE PROBLEM}

Assume a new choice of independent variables $V^{\prime}, S^{\prime}$ (it will henceforth be assumed that the chemical variables, $N_{i}$, remain unchanged), that is, mode of description. On physical grounds, there exists a transformation, $\mathbf{t}$, and its inverse, $\mathbf{t}^{-1}$, such that

$$
\begin{aligned}
& (V, S) \rightarrow\left(V^{\prime} S^{\prime},\right)=\mathbf{t}(V, S) \\
& \left(V^{\prime}, S^{\prime}\right) \rightarrow(V, S)=\mathbf{t}^{-1}\left(V^{\prime}, S^{\prime}\right)
\end{aligned}
$$


The new variables defined by $\mathbf{t}$ :

$$
\begin{aligned}
& V^{\prime}=f(V, S) \\
& S^{\prime}=g(V, S)
\end{aligned}
$$

are functionally independent and are therefore soluble for the old variables as functions of the new.

The functions $E, H, F, G$, and formulas $C$ will transform into functions of the new coordinates, which will in general take on new functional forms. The two modes of description are completely equivalent and under the transformation, $t$, formulas must transform into identities in the new variables.

Anticipating later results, let it be assumed that under the transformation, $\boldsymbol{t}$, on the physical variables

$$
\left(V^{\prime}, S^{\prime}\right)=\mathbf{t}(V, S)
$$

and the associated transformation, $\mathrm{T}$, on the functions $E, H, F, G$ :

$$
\left(E^{\prime} H^{\prime} F^{\prime} G^{\prime}\right)=\mathrm{T}(E H F G)
$$

the form of the fundamental equations remains invariant. Then it is clear that under the combined transformation $\mathrm{Tt}$ on the complementary sets $(E H F(T)$ and $(V S)$, the formulas $C$ also remain invariant. The problem can be formulated as follows: If the basic equations of thermodynamics are written in terms of a particular set of physical variables of state, what transformation on the dependent functions and associated transformation on the independent variables, leave the form of the equations invariant? If it should happen that the combined transformations $\mathrm{Tt}$ constitute a group $\mathrm{G}$, fundamental formulas will remain invariant under $\mathbf{G}$. All modes of description associated to $\mathbf{G}$ are equivalent and the group $\mathbf{G}$ characterizes a fundamental arbitrariness in the thermodynamic description of all systems.

The problem is analogous to that encountered in the Relativity Theory. It is well known that if the electromanetic field vectors $E, H$ undergo a particular linear transformation and the independent variables (xyzt) undergo an associated linear transformation (the Lorentz-Einstein Transformation), the fundamental equations of the field remain invariant. The arbitrariness of the description (theory) is characterized by the Lorentz-Einstein Group, and all reference frames associated to the group are equivalent.

\section{TANGENTIAL TRANSFORMATIONS}

The general Tangential Transformation is defined by Lie [3] as follows:

"When $Z, x_{1} \ldots \ldots X_{n}, P_{1} \ldots P_{n}$ are $2 n+1$ independent functions of the $2 n+1$ independent quantities $z, x_{1} \ldots x_{n}$, $p_{1} \ldots p_{n}$ such that the equation

$$
\begin{gathered}
d Z-\sum_{i=1}^{n} P_{i} d X_{i}=\rho\left(d z-\sum_{i=1}^{n} p_{i} d x_{i}\right) \\
\rho \neq 0
\end{gathered}
$$


is identically satisfied, the transformation

$$
\begin{aligned}
z^{\prime} & =Z\left(z, x_{i}, p_{i}\right) \\
x_{j}^{\prime} & =X_{i}\left(z, x_{i}, p_{i}\right) \\
p_{j}^{\prime} & =P_{i}\left(z, x_{i}, p_{i}\right)
\end{aligned}
$$

is a tangential (contact) transformation."

The test of a given set $Z, X_{i}, P_{i}$ is provided by Lie's Theorem [3]:

"The necessary and sufficient conditions that

$$
d Z-\sum_{i=1}^{n} P_{i} d X_{i}=\rho\left(d z-\sum_{i=1}^{n} p_{i} d x_{i}\right)
$$

be identically satisfied are

$$
\begin{array}{lc}
{\left[Z X_{i}\right]=\left[X_{i} X_{j}\right]=\left[P_{i} X_{j}\right]=\left[P_{i} P_{j}\right]=0} \\
{\left[P_{i} X_{i}\right]=\rho \quad \rho\left[Z P_{i}\right]=-\rho P_{i},}
\end{array}
$$

provided $Z, X_{i}, P_{i}$ are functionally independent, and

$$
\rho=\frac{\partial Z}{\partial z}-\sum_{i=1}^{n_{1}} P_{i} \frac{\partial X_{i}}{\partial z} \neq 0
$$

Conversely, $\rho \neq 0$ is sufficient for the functional independence of $Z$, $X_{i}, P_{i}$."

The bracket symbol is defined by

$$
[f g]=\sum_{i=1}^{n_{1}}\left(\frac{d f}{d x_{i}} \frac{\partial g}{\partial p_{i}}-\frac{d g}{d x_{i}} \frac{\partial f}{\partial p_{i}}\right) .
$$

The equations which suffice to determine the $Z, X_{i}, P_{i}, \rho$ are $2 n+1$ in number. Hence there will be an arbitrary element in solution unless some further condition is imposed upon the system. If $Z$ is specified, the $X_{i}, P_{i}$ are completely determined. The class of Cylindrical Tangential Transformations is defined by the restriction that the $X_{i}, P_{i}$ are independent of $z$. Application of the conditions of Lie's Theorem then requires that

$$
Z=\rho z+\phi\left(x_{i}, p_{i}\right),
$$

where $\rho$ is a constant.

For the case $n=3$, the conditions of Lie's Theorem are not violated if we choose

Then

$$
z=f(x y) \quad p_{1}=\frac{\partial z}{\partial x}=z_{x} \quad p_{2}=\frac{\partial z}{\partial y}=z_{y} .
$$

$$
d z^{\prime}-p_{1}{ }^{\prime} d x^{\prime}-p_{2}{ }^{\prime} d y^{\prime} \equiv \rho\left(d z-z_{x} d x-z_{y} d y\right) \equiv 0,
$$

and if $z^{\prime}$ is chosen as a function of $z, x, y, z_{x}, z_{y}$ the variables $p_{1}{ }^{\prime}, p_{2}{ }^{\prime}$, $x^{\prime}, y^{\prime}$ are determined. The transformation insures the exactness of $d z^{\prime}$, and hence $z^{\prime}$ plays the same role in the primed coordinates as $z$ does in the unprimed. 


\section{THE LEGENDRE TRANSFORMATIONS}

The thermodynamic transformations between the four equivalent modes of description in the sets of variables $(V, S),(P, S),(V, T),(P, T)$ are related to the three Legendreian Cylindrical Tangential Transformations defined by $\left(Z_{1 \times 1}=\partial Z_{1} / \partial x_{1}\right.$, etc $)$ :

$$
\begin{array}{ll}
\text { (a) } & Z_{2}=Z_{1}-Z_{1 x_{1}} x_{1} \\
\text { (b) } & Z_{3}=Z_{1}-Z_{11_{1}} y_{1} \\
\text { (c) } & Z_{4}=Z_{1}-Z_{1 v_{1}} y_{1}=Z_{2}+Z_{3}-Z_{1} .
\end{array}
$$

Applying the fundamental theorem, it is found that solutions for these cases are
(a)

$$
Z_{2}=Z_{1}-Z_{1 x_{1}} x_{1}
$$

$$
\begin{aligned}
Z_{2 x_{2}} & =x_{1} \\
x_{2} & =-Z_{1 x_{1}}
\end{aligned}
$$$$
Z_{2 \nu_{2}}=Z_{1 \nu_{1}}
$$$$
\rho=1
$$
(b) $\quad Z_{3}=Z_{1}-Z_{1 y_{1}} y_{1}$

$$
\begin{gathered}
Z_{3 x_{3}}=Z_{1 x_{1}} \\
x_{3}=x_{1}
\end{gathered}
$$$$
y_{2}=y_{1}
$$$$
\text { p }
$$
(c)

$$
\begin{aligned}
Z_{4}=Z_{1}-Z_{1 x_{1}} x_{1}-Z_{1 y_{1}} y_{1} Z_{4 x_{4}} & =x_{1} & Z_{4 y_{4}} & =-y_{1} \\
x_{4} & =-Z_{1 x_{1}} & y_{4} & =Z_{1 v_{1}}
\end{aligned}
$$$$
Z_{3 \nu_{3}}=-y_{1}
$$$$
y_{3}=Z_{1 v_{1}}
$$$$
\rho=1
$$$$
Z_{4 y_{4}}=-y_{1}
$$$$
\rho=1
$$

Under these transformations:

(a)

$$
\begin{array}{cccc}
Z_{1}=Z_{2}-Z_{2 x_{2} x_{2}} & Z_{1 x_{1}}=-x_{2} & Z_{1 \nu_{1}}=Z_{2 \nu_{2}} & \\
& x_{1}=Z_{2 x_{2}} & { }_{\nu 1}=y_{2} & \\
Z_{1}=Z_{3}-Z_{3 \nu_{3}} y_{3} & Z_{1 x_{1}}=Z_{3 x_{3}} & Z_{1 \nu_{1}}=y_{3} & \\
& x_{1}=x_{3} & y_{1}=-Z_{1 \nu_{3}} & \rho=1
\end{array}
$$

(c)

$$
\begin{aligned}
Z_{1}=Z_{4}-Z_{4 x_{4}} x_{4}-Z_{4 y_{4}} y_{4} Z_{1 x_{1}}=-x_{4} \quad Z_{1 v_{1}}=y_{4} \\
x_{1}=Z_{4 x_{4}} \quad y_{1}=-Z_{4 v_{4}} \quad \rho=1
\end{aligned}
$$

It is to be observed that:

1. The form of the equation defining the Legendre function is invariant under the transformation.

2. The transformations are involutory, that is, applied twice in succession they generate the identity transformation.

3. The Legendre functions defining the inverse transformations can be obtained by a simple substitution. Thus if:

$$
\begin{array}{ll}
Z_{1} \leftrightarrow Z_{i} & \left(\left|Z_{1 x}\right|,\left|x_{1}\right|\right) \leftrightarrow\left(\left|Z_{i x_{i}}\right|,\left|x_{i}\right|\right) \\
& \left(\left|Z_{1 y}\right|,\left|y_{1}\right|\right) \leftrightarrow\left(\left|Z_{i y_{i}}\right|,\left|y_{i}\right|\right)
\end{array}
$$

then equations $2 \leftrightarrow 3$.

The absolute values $\left|x_{i}\right|,\left|y_{i}\right|,\left|Z_{i x_{i}}\right|,\left|Z_{i y_{i}}\right|$ must be taken in order to compensate for the change in sign occasioned by transposition when the equations are solved in the usual fashion. 
The three simple substitutions carrying equations 2 into 3 can also be considered as linear transformations on complementary sets of quantities. Those on $x_{1}, y_{1}, Z_{1 x_{1}}, Z_{1 y_{1}}$, including the identity, can be conveniently written in matrix form. They are

$$
\begin{array}{cc}
\left(\begin{array}{c}
x_{1} \\
-y_{1} \\
Z_{1 x_{1}} \\
Z_{1 y_{1}}
\end{array}\right)=\mathbf{t}_{11}\left(\begin{array}{c}
x_{1} \\
-y_{1} \\
Z_{1 x_{1}} \\
Z_{1 v_{1}}
\end{array}\right) \quad\left(\begin{array}{c}
-x_{2} \\
-y_{2} \\
Z_{2 x_{2}} \\
Z_{2 y_{2}}
\end{array}\right)=\mathbf{t}_{21}\left(\begin{array}{c}
x_{1} \\
-y_{1} \\
Z_{1 x_{1}} \\
Z_{1 v_{1}}
\end{array}\right) \\
\left(\begin{array}{l}
x_{3} \\
y_{3} \\
Z_{3 x_{3}} \\
Z_{3 y_{3}}
\end{array}\right)=\mathbf{t}_{31}\left(\begin{array}{c}
x_{1} \\
-y_{1} \\
Z_{1 x_{1}} \\
Z_{1 y_{1}}
\end{array}\right) \quad\left(\begin{array}{rr}
-x_{4} \\
y_{4} \\
Z_{4 x_{4}} \\
Z_{4 y_{4}}
\end{array}\right)=\mathbf{t}_{41}\left(\begin{array}{c}
x_{1} \\
-y_{1} \\
Z_{1 x_{1}} \\
Z_{1 v_{1}}
\end{array}\right) ; \\
\mathbf{t}_{11}=\left(\begin{array}{llll}
1 & 0 & 0 & 0 \\
0 & 1 & 0 & 0 \\
0 & 0 & 1 & 0 \\
0 & 0 & 0 & 1
\end{array}\right) \quad \mathbf{t}_{21}=\left(\begin{array}{llll}
0 & 0 & 1 & 0 \\
0 & 1 & 0 & 0 \\
1 & 0 & 0 & 0 \\
0 & 0 & 0 & 1
\end{array}\right) \quad \mathbf{t}_{31}=\left(\begin{array}{llll}
1 & 0 & 0 & 0 \\
0 & 0 & 0 & 1 \\
0 & 0 & 1 & 0 \\
0 & 1 & 0 & 0
\end{array}\right) \mathbf{t}_{41}=\left(\begin{array}{llll}
0 & 0 & 1 & 0 \\
0 & 0 & 0 & 1 \\
1 & 0 & 0 & 0 \\
0 & 1 & 0 & 0
\end{array}\right)
\end{array}
$$

The matrices $t_{i 1}$ are operators which carry the space $\left(x_{1},-y_{1}, Z_{1 x_{1}}\right.$, $Z_{1 v_{1}}$ ) into any of its equivalents. They also represent simple permutation operators and can be written

$$
\mathbf{t}_{11}=\mathbf{P}(I) E \quad \mathbf{t}_{21}=\mathbf{P}(13) E \quad \mathbf{t}_{31}=\mathbf{P}(24) E \quad \mathbf{t}_{41}=\mathbf{P}(13)(24) E,
$$

where $E$ is the unit matrix $\left(\mathbf{t}_{11}\right)$ and the $P$ 's are the permutations on the rows of $E$ or the fundamental (1 space).

It can be readily verified that the $\mathbf{t}_{i 1}$ represent an Abelian group $\mathbf{t}$ of transformations isomorphic with the four element permutation group $\mathbf{P}(1)(2)(3)(4) ; \mathbf{P}(13)(2)(4) ; \mathbf{P}(1)(3)(24) ; \mathbf{P}(13)(24)$. These groups of operators transform the equivalent spaces characteristic to $Z_{1}, Z_{2}, Z_{3}, Z_{4}$ into each other. They have the multiplication tables:

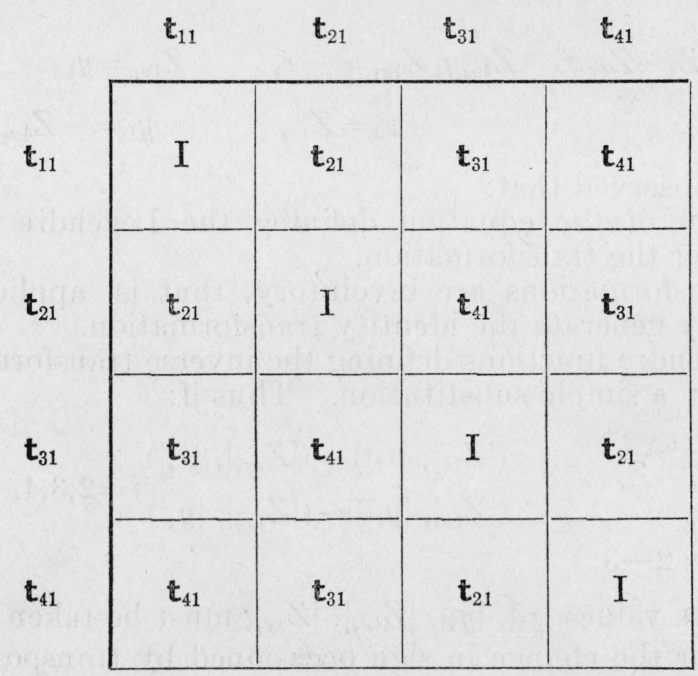




\begin{tabular}{|l|c|c|c|c|}
\multicolumn{1}{|l}{$\mathbf{P}(\mathbf{I})$} & $\mathbf{P}(13)$ & $\mathbf{P}(24)$ & $\mathbf{P}(13)(24)$ \\
\hline $\mathbf{P}(\mathbf{I})$ & $\mathbb{I}$ & $\mathbf{P}(13)$ & $\mathbf{P}(24)$ & $\mathbf{P}(13)(24)$ \\
\hline $\mathbf{P}(13)$ & $\mathbf{P}(13)$ & $\mathbb{I}$ & $\mathbf{P}(13)(24)$ & $\mathbf{P}(24)$ \\
\hline $\mathbf{P}(24)$ & $\mathbf{P}(24)$ & $\mathbf{P}(13)(24)$ & $\mathbb{I}$ & $\mathbf{P}(13)$ \\
\hline $\mathbf{P}(13)(24)$ & $\mathbf{P}(13)(24)$ & $\mathbf{P}(24)$ & $\mathbf{P}(13)$ & $\mathbf{I}$ \\
\hline
\end{tabular}

Written in terms of the space $\left(x_{1}-y_{1} Z_{1 x_{1}} Z_{1 y_{1}}\right)$, the permutation group $\mathbf{g}$ contains the elements

$$
\begin{array}{ll}
\mathbf{g}_{1}=\left(x_{1}\right)\left(-y_{1}\right)\left(Z_{1 x_{1}}\right)\left(Z_{1 v_{1}}\right) & \mathbf{g}_{2}=\left(x_{1} Z_{1 x_{1}}\right)\left(-y_{1}\right)\left(Z_{1 v_{1}}\right) \\
\mathbf{g}_{3}=\left(x_{1}\right)\left(Z_{1 x_{1}}\right)\left(-y_{1} Z_{1 v_{1}}\right) & \mathbf{g}_{4}=\left(x_{1} Z_{1 x_{1}}\right)\left(-y_{1} Z_{1 v_{1}}\right)
\end{array}
$$

This group is isomorphic to a group of permutations on the vertices of a square which leave the square invariant. In the figure

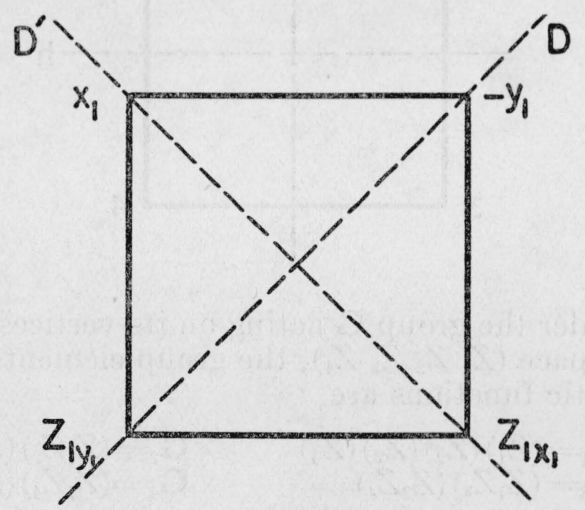

the transformations, $\mathbf{t}_{i 1}$, or permutation operators, $\mathbf{g}_{i}$, have the following correspondence to the ordinary symmetry operations

$$
\mathrm{g}_{1}=\mathrm{I} \quad \mathrm{g}_{2}=\mathrm{D} \quad \mathrm{g}_{3}=\mathrm{D}^{\prime} \quad \mathrm{g}_{4}=\mathrm{R}^{\prime \prime},
$$

where $D, D^{\prime}$ are reflections in the diagonal planes, $R^{\prime \prime}$ is a rotation of $180^{\circ}$, and $I$ is the identity operation (except for notation this group comprises the $V,-S,-P, T$ part of figure $1(\mathrm{a}), 1(\mathrm{~g}), 1(\mathrm{e}), 1(\mathrm{c})$ of Koenig's paper). 
If equations $1^{\prime}$, as a group, are considered in a standard form, then it is easily shown that this form remains invariant under the group of contact transformations, $\mathbf{t}_{i 1}$, provided the functions $Z_{1}, Z_{2}, Z_{3}, Z_{4}$, when they occur explicitly are subjected to the associated transformations

$$
\begin{gathered}
\left(\begin{array}{l}
Z_{1} \\
Z_{2} \\
Z_{3} \\
Z_{4}
\end{array}\right)=\mathbf{T}_{11}\left(\begin{array}{l}
Z_{1} \\
Z_{2} \\
Z_{3} \\
Z_{4}
\end{array}\right)\left(\begin{array}{l}
Z_{2} \\
Z_{1} \\
Z_{4} \\
Z_{3}
\end{array}\right)=\mathbf{T}_{21}\left(\begin{array}{l}
Z_{1} \\
Z_{2} \\
Z_{3} \\
Z_{4}
\end{array}\right)\left(\begin{array}{l}
Z_{3} \\
Z_{4} \\
Z_{1} \\
Z_{2}
\end{array}\right)=\mathbf{T}_{31}\left(\begin{array}{l}
Z_{1} \\
Z_{2} \\
Z_{3} \\
Z_{4}
\end{array}\right)\left(\begin{array}{l}
Z_{4} \\
Z_{3} \\
Z_{2} \\
Z_{1}
\end{array}\right)=\mathrm{T}_{41}\left(\begin{array}{l}
Z_{1} \\
Z_{2} \\
Z_{3} \\
Z_{4}
\end{array}\right) \\
\mathbf{T}_{11}=\left(\begin{array}{llll}
1 & 0 & 0 & 0 \\
0 & 1 & 0 & 0 \\
0 & 0 & 1 & 0 \\
0 & 0 & 0 & 1
\end{array}\right) \mathbf{T}_{21}=\left(\begin{array}{llll}
0 & 1 & 0 & 0 \\
1 & 0 & 0 & 0 \\
0 & 0 & 0 & 1 \\
0 & 0 & 1 & 0
\end{array}\right) \mathbf{T}_{31}=\left(\begin{array}{llll}
0 & 0 & 1 & 0 \\
0 & 0 & 0 & 1 \\
1 & 0 & 0 & 0 \\
0 & 1 & 0 & 0
\end{array}\right) \mathbf{T}_{41}=\left(\begin{array}{llll}
0 & 0 & 0 & 1 \\
0 & 0 & 1 & 0 \\
0 & 1 & 0 & 0 \\
1 & 0 & 0 & 0
\end{array}\right)
\end{gathered}
$$

These matrices represent permutation operators on the rows of the unit matrix, or on the space $\left(\begin{array}{llll}Z_{1} & Z_{2} & Z_{3} & Z_{4}\end{array}\right)$

$$
\begin{aligned}
\mathbf{T}_{11} \sim \mathbf{P}(1)(2)(3)(4)=\mathbf{G}_{1} & \mathbf{T}_{21} \sim \mathbf{P}(12)(34)=\mathbf{G}_{2} \\
\mathbf{T}_{31} \sim \mathbf{P}(13)(24)=\mathbf{G}_{3} & \mathbf{T}_{41} \sim \mathbf{P}(14)(23)=\mathbf{G}_{4}
\end{aligned}
$$

The $\mathbf{T}_{i 1}$ constitute an Abelian group isomorphic to the permutation group $\mathbf{G}$ composed of the $\mathbf{G}_{\imath}$. The square

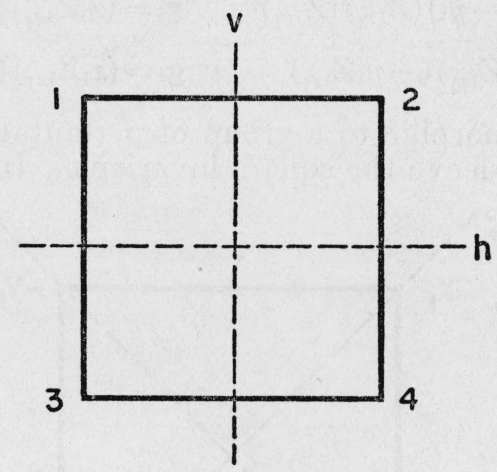

is invariant under the group $\mathbf{G}$ acting on its vertices. Relabeling the figure for the space $\left(Z_{1} Z_{2} Z_{3} Z_{4}\right)$, the group elements of $\mathbf{G}$ in terms of the characteristic functions are

$$
\begin{array}{ll}
\mathbf{G}_{1}=\left(Z_{1}\right)\left(Z_{2}\right)\left(Z_{3}\right)\left(Z_{4}\right) & \mathbf{G}_{2}=\left(Z_{1} Z_{2}\right)\left(Z_{3} Z_{4}\right) \\
\mathbf{G}_{3}=\left(Z_{1} Z_{3}\right)\left(Z_{2} Z_{4}\right) & \mathbf{G}_{4}=\left(Z_{1} Z_{4}\right)\left(Z_{2} Z_{3}\right)
\end{array}
$$

These elements correspond, in order, to the following symmetry operations:

$I=$ Identity.

$H=$ Reflection in $h$.
$V=$ reflection in $v$. $R^{\prime \prime}=$ Rotation of $180^{\circ}$. 
and have the multiplication table:

\begin{tabular}{|c|c|c|c|c|}
\hline & $\begin{array}{c}\mathbf{G}_{1} \\
\mathbf{G}_{1}\end{array} \mathbf{G}_{2}$ & $\mathbf{G}_{3}$ & $\mathbf{G}_{4}$ \\
\hline $\mathbf{I}$ & $\mathbf{G}_{2}$ & $\mathbf{G}_{3}$ & $\mathbf{G}_{4}$ \\
\hline $\mathbf{G}_{2}$ & $\mathbf{G}_{2}$ & $\mathbf{I}$ & $\mathbf{G}_{4}$ & $\mathbf{G}_{3}$ \\
\hline $\mathbf{G}_{3}$ & $\mathbf{G}_{3}$ & $\mathbf{G}_{4}$ & $\mathbf{I}$ & $\mathbf{G}_{2}$ \\
\hline $\mathbf{G}_{4}$ & $\mathbf{G}_{4}$ & $\mathbf{G}_{3}$ & $\mathbf{G}_{2}$ & $\mathbf{I}$ \\
\hline
\end{tabular}

The preceding results can be condensed into:

Theorem 1.-The fundamental equations $1^{\prime}$, remain invariant under the Contact Transformations defined by equations 2 and the associated group $\mathbf{G}$ on the Legendre transformation functions, provided absolute values are taken for the elements of all equivalent spaces $\left(x_{i} y_{i} Z_{1 x_{1}} Z_{i y_{1}}\right)$.

The restriction to absolute values can be removed if in applying $\mathbf{g}$ and $\mathbf{G}$ to equations $\mathbf{1}^{\prime}$, numerator and denominator of the partial derivatives are transformed independently, that is, formally. This procedure eliminates the use of the contact transformations.

The spaces of $\mathbf{G}$ and $\mathbf{g}$ are exclusive, and hence the elements $\mathbf{G}_{i} \mathbf{g}_{i}$ constitute a group $\mathscr{G}$ for the combined space $\left(Z_{1} Z_{2} Z_{3} Z_{4}\right)\left(x_{1}-y_{1}\right.$ $\left.Z_{1 x_{1}} Z_{1 v_{1}}\right)$. Consequently, Theorem 1 can be written in greater generality:

Theorem $1^{\prime}$.- The fundamental equations $1^{\prime}$ are invariant under the substitution group $\mathscr{G}$ on the space $\left(Z_{1} Z_{2} Z_{3} Z_{4}\right)\left(x_{1}-y_{1} Z_{1 x_{1}} Z_{1 v_{1}}\right)$.

In Theorem $1^{\prime}$, except for notation, $\mathscr{G}$ consists of elements $S_{1}, S_{3}$, $S_{5}, S_{7}$ of the Koenig group.

The group $\mathscr{G}$ can also be generated geometrically. It is only necessary to inscribe the square for $\mathbf{G}$ within that for $\mathbf{g}$ such that the symmetry planes $v$ and $D, b$ and $D^{\prime}$, coincide. 
There results:

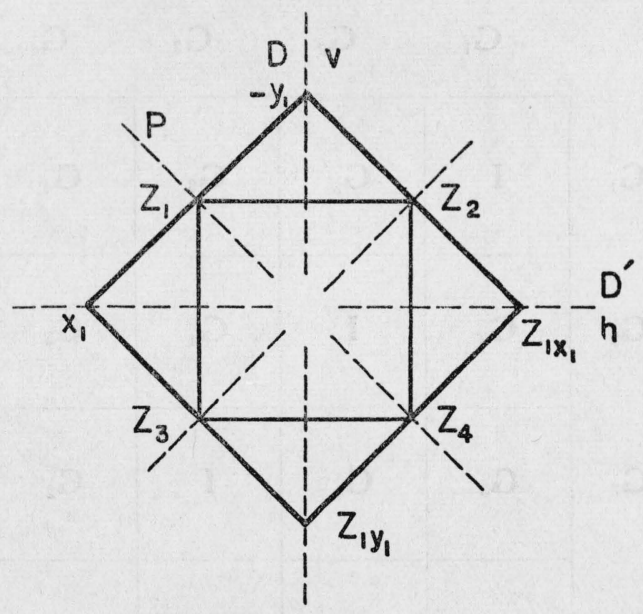

Writing out the fundamental equations 2 and associated contact transformations in the remaining coordinate systems, the following theorems are immediately evident.

Theorem 2.- The matrix operators, $\mathrm{T}_{i j}$ and $\mathrm{t}_{i j}$, which, acting on the function and coordinate space of one representation, transform the fundamental equations 2 to any other representation with invariant form, are independent of the coordinate system.

Theorem 3.-If $\mathbf{t}_{i 1}$ denotes the matrix of a Contact Transformation in a given representation, then the corresponding transformation for any other representation is given by the transform $\mathbf{t}^{-1} \mathbf{t}_{i 1} \mathbf{t}$ (or $\mathbf{t t}_{i} \mathbf{t}^{-1}$ ), where $\mathbf{t}$ corresponds to the contact transformation carrying the old to the new representation.

Theorem 4.-The permutation group $\mathscr{G}$, which leaves the fundamental equations 2 invariant is independent of the coordinate system. (Theorem 4 is a restatemet of theorem 2.)

The invariance of the four contact transformations (including the identity) defined by equations $1^{\prime}$ under the group of linear transformations, $\mathbf{t}_{i 1}$ can be extended to invariance under the substitution group $\mathscr{G}$ provided a supplementary rule of signs is introduced. For general consistency a particular equation must transform into the same equation whether directly under the substitution operator $\mathscr{G}_{i}$ or as an element of a matrix equation under the corresponding $\mathbf{t}_{i 1}$. The equation

$$
Z_{2 x_{2}}=x_{1}
$$

transforms under $\boldsymbol{t}_{41}$ as an element of

$$
\left(\begin{array}{c}
-x_{2} \\
-y_{2} \\
Z_{2 x_{2}} \\
Z_{2 y_{2}}
\end{array}\right)=\operatorname{c}_{21}\left(\begin{array}{c}
x_{1} \\
-y_{1} \\
Z_{1 x_{1}} \\
Z_{1 y_{1}}
\end{array}\right)
$$


and therefore becomes

$$
\begin{gathered}
\mathbf{t}_{41}\left(\begin{array}{c}
-x_{2} \\
-y_{2} \\
Z_{2 x_{2}} \\
Z_{2 v_{2}}
\end{array}\right)=\mathbf{t}_{41} \mathbf{t}_{21}\left(\begin{array}{c}
x_{1} \\
-y_{1} \\
Z_{1 x_{1}} \\
Z_{1 v_{1}}
\end{array}\right)=\mathbf{t}_{31}\left(\begin{array}{c}
x_{1} \\
-y_{1} \\
Z_{1 x_{1}} \\
Z_{1 v_{1}}
\end{array}\right)=\left(\begin{array}{c}
x_{3} \\
y_{3} \\
Z_{3 x_{3}} \\
Z_{3 y_{2}}
\end{array}\right) \\
\left(Z_{2 x_{2}}=x_{1}\right) \rightarrow\left(Z_{3 x_{3}}=Z_{\left.1 x_{1}\right)}\right) .
\end{gathered}
$$

Before the substitution operator $\mathscr{G}_{4}$ can be applied to the given equation, the left side must be transformed into the space characteristic of $\mathscr{G}$, that is, (1 space). Hence

$$
\frac{\partial Z_{2}}{\partial x_{2}}=\frac{\partial\left(\mathbf{T}_{21} Z_{1}\right)}{\partial\left(\mathbf{t}_{21} x_{1}\right)}=\frac{\partial\left(\mathbf{G}_{2} Z_{1}\right)}{\partial\left(-Z_{1 x_{1}}\right)}=x_{1}
$$

where $\mathbf{T}_{2 i} Z_{1}$ and $\mathbf{t}_{21} x_{1}$ denote the matrix transforms of $Z_{2}$ and $x_{2}$. Applying $\mathscr{G}_{4}$.

$$
\begin{gathered}
\mathscr{G}_{1}\left(\frac{\partial Z_{2}}{\partial x_{2}}\right)=\frac{\partial\left(\mathbf{G}_{4} \mathbf{G}_{2}\right) Z_{1}}{-\partial\left(\mathbf{g}_{4} Z_{1 x_{1}}\right)}=\frac{\partial\left(\mathbf{G}_{3} Z_{3}\right)}{-\partial x_{1}}=\frac{\partial Z_{3}}{-\partial x_{1}}=\frac{\partial Z_{3}}{-\partial x_{3}} \\
\mathscr{G}_{4} x_{1}=Z_{1 x_{1}}
\end{gathered}
$$

and consequently

$$
\left(\frac{\partial Z_{2}}{\partial x_{2}}=x_{1}\right) \rightarrow\left(\frac{\partial Z_{3}}{-\partial x_{3}}=Z_{1 x_{1}}\right)
$$

which is incorrect in sign on the left side. This alternation in sign will always occur when the transform of $x_{i}$ and its conjugate $Z_{1 x_{1}}$ differ in sign. Hence the rule:

Rule of Signs.-If $x_{i}$ and $Z_{i x_{i}}$ of a given space transform with opposite signs, then in transforming $Z_{i x_{i}}$ by substitution, it must be replaced by $Z_{i\left|x_{i}\right|}$. If, of course, $x_{i}$ and $Z_{i x_{i}}$ transform with the same sign, then the rule is unnecessary, but if applied, will yield correct results, provided $Z_{i x_{i}}$ always transforms positive. This is in fact the case. There results:

Theorem 5.-The Contact Transformations associated with the fundamental equations 2 in any coordinate system are invariant under the substitution group $\mathscr{G}$ characteristic of that coordinate system, provided $Z_{i x_{i}}$ is replaced by $Z_{i\left|x_{i}\right|}$. (This is the origin of Koenig's Rule of Signs.)

If equations $1^{\prime}$ are invariant under an extended group $\mathscr{G}^{*}$, La. granges' Theorem on the decomposition of a finite group insures the order of this group to be a multiple of the order of $\mathscr{G}$, that is, 4 . The order of $\mathscr{G}^{*} \leqq 24$, the order of the complete symmetric group on four elements, and therefore $\mathscr{G} *$ if it exists, must be of order 8 or 12 . The group $\mathscr{G}^{*}$ will include $\mathscr{G}$ and its cosets $\mathscr{G} \mathbf{g}_{i}\left(\right.$ or $\left.\mathbf{g}_{i} \mathscr{G}\right)$, where $\mathbf{g}_{i}=$ $\mathbf{S}_{i} \mathbf{s}_{i}$ is an element of $\mathscr{G}^{*}$ not in $\mathscr{G}$ and $\mathbf{S}_{i}, \mathbf{s}_{i}$ operate on $\left(Z_{1} Z_{2} Z_{3} Z_{4}\right)$ and $\left(x_{1}-y_{1} Z_{1 x_{1}} Z_{1 v_{1}}\right)$, respectively. 
Examination of equations $1^{\prime}$ show the basic form to be invariant under the symmetry operator

$$
\mathbf{g}^{\prime}=\left(Z_{1}\right)\left(Z_{4}\right)\left(Z_{2} Z_{3}\right)\left(x_{1}-y_{1}\right)\left(Z_{1 x_{1}} Z_{1 y_{1}}\right),
$$

which corresponds geometrically to reflection of the generating square in the plane $\mathbf{P}$ ( $\mathbf{g}^{\prime}$ corresponds to $S_{6}$ of Koenig's group). The elements of the coset $\mathscr{G} \mathbf{g}^{\prime}$ are

$$
\begin{aligned}
& \mathscr{G}_{5}=\mathscr{G}_{1} \mathbf{g}^{\prime}=\left(Z_{1}\right)\left(Z_{4}\right)\left(Z_{2} Z_{3}\right)\left(x_{1}-y_{1}\right)\left(Z_{1 x_{1}} Z_{1 v_{1}}\right) \\
& \mathscr{G}_{6}=\mathscr{G}_{2} \mathbf{g}^{\prime}=\left(Z_{1} Z_{2} Z_{4} Z_{3}\right)\left(x_{1}-y_{1} Z_{1 x_{1}} Z_{1 \nu_{1}}\right) \\
& \mathscr{G}_{7}=\mathscr{G}_{3} \mathbf{g}^{\prime}=\left(Z_{1} Z_{3} Z_{4} Z_{2}\right)\left(x_{1} Z_{v_{1}} Z_{x_{1}}-y_{1}\right) \\
& \mathscr{G}_{8}=\mathscr{G}_{4} \mathbf{g}^{\prime}=\left(Z_{1} Z_{4}\right)\left(Z_{2}\right)\left(Z_{3}\right)\left(x_{1} Z_{1 \nu_{1}}\right)\left(-y_{1} Z_{1 x_{1}}\right)
\end{aligned}
$$

They do not form a group and do not correspond to contact transforma-

\begin{tabular}{|c|c|c|c|c|c|c|c|}
\hline $\mathscr{G}_{1}$ & $\mathscr{G}_{2}$ & $\mathscr{G}_{3}$ & $\mathscr{G}_{4}$ & $\mathscr{G}_{5}$ & $\mathscr{G}_{6}$ & $\mathscr{G}_{7}$ & $\mathscr{G}_{8}$ \\
\hline $\mathscr{G}_{1}$ & $\mathscr{G}_{2}$ & $\mathscr{G}_{3}$ & $\mathscr{G}_{4}$ & $\mathscr{G}_{5}$ & $\mathscr{G}_{6}$ & $\mathscr{G}_{7}$ & $\mathscr{G}_{8}$ \\
\hline $\mathscr{G}_{2}$ & $\mathscr{I}$ & $\mathscr{G}_{4}$ & $\mathscr{G}_{3}$ & $\mathscr{G}_{6}$ & $\mathscr{G}_{5}$ & $\mathscr{G}_{8}$ & $\mathscr{G}_{7}$ \\
\hline $\mathscr{G}_{3}$ & $\mathscr{G}_{4}$ & $\mathscr{I}$ & $\mathscr{G}_{2}$ & $\mathscr{G}_{7}$ & $\mathscr{G}_{8}$ & $\mathscr{G}_{5}$ & $\mathscr{G}_{6}$ \\
\hline $\mathscr{G}_{4}$ & $\mathscr{G}_{3}$ & $\mathscr{G}_{2}$ & $\mathscr{I}$ & $\mathscr{G}_{8}$ & $\mathscr{G}_{7}$ & $\mathscr{G}_{6}$ & $\mathscr{G}_{5}$ \\
\hline $\mathscr{G}_{5}$ & $\mathscr{G}_{7}$ & $\mathscr{G}_{6}$ & $\mathscr{G}_{8}$ & $\mathscr{I}$ & $\mathscr{G}_{3}$ & $\mathscr{G}_{2}$ & $\mathscr{G}_{4}$ \\
\hline $\mathscr{G}_{6}$ & $\mathscr{G}_{8}$ & $\mathscr{G}_{5}$ & $\mathscr{G}_{7}$ & $\mathscr{G}_{2}$ & $\mathscr{G}_{4}$ & $\mathscr{I}$ & $\mathscr{G}_{3}$ \\
\hline $\mathscr{G}_{7}$ & $\mathscr{G}_{5}$ & $\mathscr{G}_{8}$ & $\mathscr{G}_{6}$ & $\mathscr{G}_{3}$ & $\mathscr{I}$ & $\mathscr{G}_{4}$ & $\mathscr{G}_{2}$ \\
\hline $\mathscr{G}_{8}$ & $\mathscr{G}_{6}$ & $\mathscr{G}_{7}$ & $\mathscr{G}_{5}$ & $\mathscr{G}_{4}$ & $\mathscr{G}_{2}$ & $\mathscr{G}_{3}$ & $\mathscr{I}$ \\
\hline
\end{tabular}
tions. They may be considered as formal symmetry operators.

It is easily verified that the eight elements $\mathscr{G}_{i}$ constitute a group $\mathscr{G}^{*}$ which leaves the generating square invariant. It is therefore isomorphic to the Octic Group and has the multiplication table:

The general result can be incorporated in theorem 6 . 
Theorem 6.-The form of the fundamental equations $1^{\prime}$ is invariant under the group $\mathscr{G} *$, and this group is the largest permutation group having this property.

An additional proof that $\mathscr{G}^{*}$ is the largest permutation group which preserves the standard form lies in the fact that the Octic Group is the largest permutation group under which the bilinear form $\left(x_{1}+x_{2}-x_{3}-x_{4}\right)^{2}$ is invariant. The last of equations $1^{\prime}$ can be written

$$
\left(Z_{1}+Z_{4}-Z_{2}-Z_{3}\right)^{2}=0,
$$

and this must be true under all transformations. Except for notation, this is the bilinear form invarient under the Octic Group. Hence the theorem.

The matrix representatives of the new operators $g_{5} \ldots \mathbf{g}_{8}$ are

$$
\mathbf{t}_{51}=\left(\begin{array}{llll}
0 & 1 & 0 & 0 \\
1 & 0 & 0 & 0 \\
0 & 0 & 0 & 1 \\
0 & 0 & 1 & 0
\end{array}\right) \quad \mathbf{t}_{61}=\left(\begin{array}{llll}
0 & 1 & 0 & 0 \\
0 & 0 & 1 & 0 \\
0 & 0 & 0 & 1 \\
1 & 0 & 0 & 0
\end{array}\right) \quad \mathbf{t}_{71}=\left(\begin{array}{llll}
0 & 0 & 0 & 1 \\
1 & 0 & 0 & 0 \\
0 & 1 & 0 & 0 \\
0 & 0 & 1 & 0
\end{array}\right) \quad \mathbf{t}_{81}=\left(\begin{array}{llll}
0 & 0 & 0 & 1 \\
0 & 0 & 1 & 0 \\
0 & 1 & 0 & 0 \\
1 & 0 & 0 & 0
\end{array}\right),
$$

which taken with those representing $\mathbf{g}_{1} \ldots \mathbf{g}_{4}$ for a group $\mathbf{t}^{*}$ having a multiplication table in $1-1$ correspondence with that of $\mathscr{G}^{*}$ Contact transformations are transformed under these operators exactly as previously described. For example, the transformation defined by equations $2 \mathrm{a}$ is transformed by $\mathscr{G}_{6}$ into

$$
t_{61}\left(\begin{array}{c}
2 \\
\text { space }
\end{array}\right)=t_{21} t_{61}\left(\begin{array}{c}
1 \\
\text { space }
\end{array}\right)=t_{51}\left(\begin{array}{c}
1 \\
\text { space }
\end{array}\right)
$$

which is equivalent to

$$
t_{31}\left(\begin{array}{c}
2 \\
\text { space }
\end{array}\right)=\left(\begin{array}{c}
4 \\
\text { space }
\end{array}\right)
$$

the contact transformation characteristic of $Z_{4}$ expressed in the coordinates of (2 space). Similar results are obtained for other operators. The general result is contained in theorem:

Theorem 7.-The Contact Transformations defined by equations 2 are invariant under the group $\mathbf{t}^{*}$ of elements $\mathbf{t}_{i 1} i=1 . .8$. Thus:

The preceding theorems can now be extended to the larger groups.

Theorem $2^{\prime}$.-The matrix groups $\mathrm{T}^{*}$ and $\mathbf{t}^{*}$, under which the fundamental equations $1^{\prime}$ are invariant, are independent of the representation. 
Theorem $4^{\prime}$.- The permutation group $\mathscr{G}^{*}$ which leaves the fundamental equations $1^{\prime}$ invariant is independent of the representation.

Theorem 5'.-The Contact Transformations associated with the fundamental equations 2 in any representation are invariant under the substitution group $\mathscr{G}^{*}$ characteristic of that representation provided $Z_{i x_{i}}$ is replaced by $Z_{i}\left|x_{i}\right|$.

A consequence of the invariance of the contact transformations under the substitution group $\mathscr{G}^{*}$ is the existence of characteristic families of equations also invariant under $\mathscr{G}^{*}$. Families having important correlatives in general thermodynamic theory are contained in the following theorems. Given an equation of the form expressed in the theorem, the group $\mathscr{G}^{*}$ generates the entire family, provided the contact transformations themselves are employed to reduce the symbolism.

Theorem 8.-The family of four differential forms

$$
Z_{i}-\left(\frac{\partial Z_{i}}{\partial x_{i}}\right)_{y_{i}} d x_{i}-\left(\frac{\partial Z_{i}}{\partial x_{i}}\right)_{x_{i}} d y_{i}=0
$$

is invariant under the substitution group $\mathscr{G} *$.

Theorem 9.-The eight member family of the form

$$
\left(\frac{\partial Z_{i}}{\partial x_{i}}\right)_{y_{i}}=-x_{j}
$$

is invariant under the group $\mathscr{G}^{*}$.

Theorem 10.-The four equations of the form

$$
\left(\frac{\partial Z_{i}}{\partial x_{i}}\right)_{v_{j}}=\left(\frac{\partial Z_{i}}{\partial x_{j}}\right)_{y_{i}}
$$

constitute a family invariant under the characteristic group $\mathscr{G}$ *.

Theorem 11.- The family of eight identities

$$
\frac{\partial^{2} Z_{i}}{\partial x_{i} \partial y_{i}}=\frac{\partial^{2} Z_{i}}{\partial y_{i} \partial x_{i}}
$$

is invariant under $\mathscr{G}^{*}$.

The invariance of the fundamental equations yields theorems 12 , 13, and 14:

Theorem 12.-The family of eight equations of the form

$$
Z_{i}=Z_{j}-x_{j}-\left(\frac{\partial Z_{j}}{\partial x_{j}}\right)_{y_{j}}
$$

is invariant under the group $\mathscr{G} *$.

Theorem 13.- The four equations of the form

$$
Z_{i}=Z_{j}-x_{j}\left(\frac{\partial Z_{j}}{\partial x_{j}}\right)_{y_{j}}-y_{j}\left(\frac{\partial Z_{j}}{\partial y_{j}}\right)_{x_{j}}
$$

constitute a family invariant under the group $\mathscr{G}^{*}$.

Theorem 14.-The equation

$$
\left(Z_{1}+Z_{4}\right)-\left(Z_{2}+Z_{3}\right)=0
$$


is invariant under the group $\mathscr{G}^{*}$.

Other families of formulas invariant under $\mathscr{G}^{*}$ are deducible from the fundamental equations $1^{\prime}$ by the application of operators such as $\partial / \partial x, \partial / \partial y, d$.

The transform of a formula obtained by applying the operator $\mathrm{O}$ to one of the fundamental equations is derived by applying the group operator $\mathbf{g}_{i}$ to both $\mathbf{O}$ and $f$ in the equation

$$
\mathbf{O} f=0 \text {. }
$$

For example, from the equation

or

$$
Z_{1}=Z_{3}-y_{3}\left(\frac{\partial Z_{3}}{\partial y_{3}}\right)_{x_{3}}
$$

$$
Z_{1}-Z_{3}+y_{3}\left(\frac{\partial Z_{3}}{\partial y_{3}}\right)_{x_{3}}=0
$$

the operator $\partial / \partial x_{3}$ yields

$$
\frac{\partial}{\partial x_{3}}\left\{Z_{1}-Z_{3}+y_{3}\left(\frac{\partial Z_{3}}{\partial y_{3}}\right)_{x_{3}}\right\}=0
$$

and the substitution operator $\mathscr{G}_{7}$ gives

$$
\frac{\partial}{\partial\left(\mathbf{t}_{71} x_{3}\right)} \mathscr{G}_{7}\left\{Z_{1}-Z_{3}+y_{3}\left(\frac{\partial Z_{3}}{\partial y_{3}}\right)_{x_{3}}\right\}=0
$$

where the transform $t_{71} x_{3}$ is obtained from the matrix equation

$$
\left(\begin{array}{c}
3 \\
\text { space }
\end{array}\right) \rightarrow \mathbf{t}_{31} \mathbf{t}_{71}\left(\begin{array}{c}
1 \\
\text { space }
\end{array}\right)=\mathbf{t}_{51}\left(\begin{array}{c}
4 \\
\text { space }
\end{array}\right) \text {, }
$$

so that

$$
\frac{\partial}{\partial x_{3}} \rightarrow \frac{\partial}{\partial y_{4}}
$$

and the transformed equation becomes:

$$
\frac{\partial}{\partial y_{4}}\left\{Z_{3}-Z_{4}+x_{4}\left(\frac{\partial Z_{4}}{\partial x_{4}}\right)_{v_{4}}\right\}=0 .
$$

The reversal in order of the product of the matrices $\tau_{71}$ and $t_{31}$ in the transform follows from the fact that $\mathscr{G} *$ is non-Abelian and that $\mathscr{G} *$ is written in terms of (1 space). This method yields theorem 15.

Theorem 15.-Equations of the form

$$
\left(\frac{\partial Z_{i}}{\partial y_{j}}\right)_{x_{i}}-\left(\frac{\partial Z_{k}}{\partial y_{l}}\right)_{x_{l}}=-y_{j}\left\{\left(\frac{\partial^{2} Z_{j}}{\partial y_{i}^{2}}\right)_{x_{i}}-\left(\frac{\partial^{2} Z_{l}}{\partial y_{l}^{2}}\right)_{x_{l}}\right\}=-y_{j}\left\{\frac{\partial^{2} Z_{j}}{\partial x_{i} \partial y_{i}} \frac{\partial^{2} Z_{l}}{\partial x_{l} \partial y_{l}}\right\}
$$

constitute a four-member family invariant under the substitution group $\mathscr{G}^{*}$.

Theorem 16.-The eight equations of the form

$$
\left(\frac{\partial Z_{i}}{\partial y_{j}}\right)_{x_{i}}=-y_{i}\left(\frac{\partial^{2} Z_{j}}{\partial y_{j}^{2}}\right)_{x_{i}}
$$

comprise a family invariant under the substitution group $\mathscr{G}^{*}$. 
Theorem 17.- The eight member family"of form

$$
\begin{aligned}
\left(\frac{\partial y_{i}}{\partial x_{i}}\right)_{Z_{j}} & =-\left(\frac{\partial Z_{j}}{\partial x_{i}}\right)_{y_{i}} /\left(\frac{\partial Z_{j}}{\partial y_{i}}\right)_{x_{i}} \\
& =-\frac{\left\{\left(\frac{\partial Z_{i}}{\partial x_{i}}\right)_{v_{i}}-y_{i} \frac{\partial}{\partial x_{i}}\left(\frac{\partial Z_{i}}{\partial y_{i}}\right)_{x_{i}}\right\}}{\left(\frac{\partial Z_{j}}{\partial y_{i}}\right)_{x_{i}}}
\end{aligned}
$$

is invariant under the substitution group $\mathscr{G} *$.

Transformation formulas for the second derivatives can be obtained by elementary calculation from the four sets of Contact Transformations corresponding to the four equivalent representations. They give the following theorems:

Theorem 18.-The eight equations of the type

$$
Z_{j x_{j} x_{j}}=\frac{-1}{Z_{i x_{i} x_{i}}}
$$

form a family invariant under the substitution group $\mathscr{G}^{*}$.

Theorem 19.-The family of eight equations of form

$$
Z_{j x_{j} y_{j}}=\frac{-Z_{i x_{i} \nu_{i}}}{Z_{i x_{i} x_{i}}}
$$

is invariant under the substitution group $\mathscr{G}^{*}$, provided the following Rule of Signs is applied.

Rule.-The conjugates $Z_{i x_{i}}, Z_{i y_{i}}$ must be taken with absolute signs wherever they occur in the transform of $x_{j}, y_{j}$ in the basic form. $\mathscr{G}^{*}$ is expressed in (1 space).

Theorem 20.-The eight equations of form

$$
-Z_{j y_{y} y_{j}}=\frac{Z_{i x_{i} y_{i}}^{2}-Z_{i x_{i} x_{i}} Z_{i y_{i} y_{i}}}{Z_{i x_{i} x_{i}}}
$$

constitute a family invariant under the substitution group $\mathscr{G}^{*}$.

Theorem 21.-Equations of the form

$$
Z_{j x_{j} x_{j}}=\frac{Z_{i y_{i} y_{i}}}{Z_{i x_{i} y_{i}}^{2}-Z_{i x_{i} x_{i}} Z_{i y_{i} y_{i}}}
$$

constitute an eight membered family invariant under the substitution group *.

Theorem 22.-The four equations of the type

$$
Z_{j x_{j} y_{j}}=\frac{Z_{i x_{i} y_{i}}}{Z_{i x i y_{i}}^{2}-Z_{i x_{i} x_{i}} Z_{i y_{i} v_{i}}}
$$

form a family invariant under the substitution group $\mathscr{G}^{*}$, provided the Rule of Signs given in Theorem 19 is applied.

The Legendre transformation functions characteristic of a given representation are simply related to Lie's [4] theory of a "group of 
functions." A set of $n$ functions $v_{1} \ldots v_{n}$ constitute a group of order $n$ if they satisfy the two conditions:

1. $v_{i}$ are algebraically independent.

2. Every combination $\left(v_{i} v_{j}\right)$ of the set is expressible in terms of the set and

$$
\left(v_{i} v_{j}\right)=\sum_{k}\left(\frac{\partial v_{i}}{\partial x_{k}} \frac{\partial v_{j}}{\partial p_{k}}-\frac{\partial v_{i}}{\partial p_{k}} \frac{\partial v_{j}}{\partial x_{k}}\right)
$$

Any other function $u$ which has the property that all $\left(v_{i} u\right)$ are expressible in terms of the $v_{i}$ belongs to the group. If all the $\left(v_{i} u\right)$ are zero, then $u$ is said to be in involution to the group and is called an indicial function (ausgezeichnete Funktion) of the group.

Lie [5] has given a number of theorems on the properties of a group of functions of which the two following are of particular interest in connection with the preceding results.

Theorem 25L.-A group of functions in the variables $x_{i}, p_{i}$ possesses only two properties which are invariant under all contact transformations of the form

$$
Z^{\prime}=Z+\Omega(x p) \quad x_{i}{ }^{\prime}=X_{i}(x p) \quad p_{i}{ }^{\prime}=P_{i}(x p) .
$$

The first is the order of the group and the second the number of independent indicial functions of the group.

Theorem 26L.-If the $r$ independent functions $U_{1} \ldots U_{r}$ in the variables $x_{1} \ldots x_{n}, p_{1} \ldots p_{n}$ form a group of functions of order $r$, and the functions $V_{1} \ldots V_{\tau}$ in the variables $x_{1}{ }^{\prime} \ldots x_{n}{ }^{\prime}, p_{1}{ }^{\prime} \ldots p_{n}{ }^{\prime}$ likewise a group of order $r$, then there exists a contact transformation of the form

$$
Z^{\prime}=Z+\Omega(x p) \quad x_{i}^{\prime}=X_{i}(x p) \quad p_{i}^{\prime}=P_{i}(x p),
$$

transforming $U_{1} \ldots U_{r}$ into the corresponding $V_{1} \ldots V_{r}$, if and only if, every combination $\left(U_{t} U_{j}\right)$ is expressed in terms of $U_{1} \ldots U_{r}$ in the same way as the corresponding $\left(V_{i} V_{j}\right)$ in terms of the $V_{1} \ldots$ $V_{r}$.

It is easily verified that each of the sets of Legendreian functions:

$$
\begin{array}{llll}
Z_{2} & Z_{1} & Z_{4} & Z_{2} \\
Z_{3} & Z_{4} & Z_{1} & Z_{3} \\
Z_{4} & Z_{3} & Z_{2} & Z_{1}
\end{array}
$$

characteristic of the fundamental equations $1^{\prime}$ in the four equivalent representations, constitute a group of functions according to the Lie definition. Each group is of order three and possesses one indicial function. They are

$$
f_{1}=\frac{Z_{3}-Z_{2}}{Z_{4}-Z_{2}} \quad f_{2}=\frac{Z_{4}-Z_{1}}{Z_{3}-Z_{1}} \quad f_{3}=\frac{Z_{1}-Z_{4}}{Z_{2}-Z_{4}} \quad f_{4}=\frac{Z_{2}-Z_{3}}{Z_{1}-Z_{3}},
$$

and the set can be generated by the substitution group $\mathbf{G}$ on any one of them. Hence theorem 23.

Theorem 23.-The indicial functions associated with the group of Legendreian functions characteristic of the four representations constitute a four member family invariant under the substitution subgroup $\mathbf{G}$ of $\mathscr{G}^{*}$. 
An elementary calculation shows that each of the characteristic sets given above satisfies the conditions of Lie's Theorem 26, and hence are transformable into each other by a cylindrical tangential transformation. The transformations in the representation of (1 space) are those given in equations 2 . The equivalent transformations in any other representation can be obtained by applying Theorem 3 .

\section{THERMODYNAMIC FORMULAS}

If the following correspondences are made, the preceding results are directly interpretable as general thermodynamic relations.

$$
\begin{array}{cll}
Z_{1}=E & Z_{2}=H & Z_{3}=F \quad Z_{4}=G \\
\left(x_{1}, y_{1}\right)=(V, S) & \left(x_{3}, y_{3}\right)=\left(x_{1}, Z_{1 v_{1}}\right)=(V, T) \\
\left(x_{2}, y_{2}\right)=\left(-Z_{1 x_{1}}, y_{1}\right)=(P, S) & \left(x_{4}, y_{4}\right)=\left(x_{2}, y_{3}\right)=(P, T)
\end{array}
$$

Under this correspondence equations $1^{\prime}$ transform into the fundamental equations 1 and the group $\mathscr{G}^{*}$ becomes identical with the Koenig substitution group in the representation of (1 space). The generating square coincides with figure 1(a) of Koenig's paper.

The generation of a family of formulas by the action of the substitution $\mathscr{G}^{*}$ on a basic form is of course subject to the rules of signs given in the preceding section. Moreover, since a family of formulas can be expressed either in terms of the thermodynamic functions and their derivatives, or in a reduced form in terms of $I, S, P, T$ and $E, H, F, G$, the application of a rule will be determined by the character of the basic form. The general result can be expressed in the theorems:

Theorem 24.- If a basic form is deducible from the fundamental equations 1 , or equivalent in another representation, by the action of an operator $\mathbf{O}$ followed by algebraic rearrangement, the substitution group $\mathscr{G} *$ generates an invariant family of $1,2,4$, or 8 members.

Theorem 25. - If a basic form of Theorem 24 is transformed by the use of the equations of the contact transformations, then the substitution group $\mathscr{G}^{*}$ generates an invariant family of $1,2,4$, or 8 members, provided one of each conjugate pair of variables in every term of the new basic form is enclosed in the absolute sign.

The following table is a condensation of the theorems given in the preceding section on families of equations invariant under the substitution group $\mathscr{G}^{*}$.

\begin{tabular}{c|c|c}
\hline \multicolumn{1}{c|}{ Origin } & $\begin{array}{c}\text { order of } \\
\text { family }\end{array}$ & \multicolumn{1}{c}{ Equivalent basic forms } \\
\hline Theorem 8 & 4 & $d E-\left(\frac{\partial E}{\partial V}\right)_{S} d V-\left(\frac{\partial E}{\partial S}\right)_{V} d S=0$ \\
& & $\begin{array}{l}d E+|P| d V-|T| d S=0 \\
\text { Theorem } 9\end{array}$ \\
$\left(\frac{\partial E}{\partial|V|}\right)_{S}=-P$.
\end{tabular}




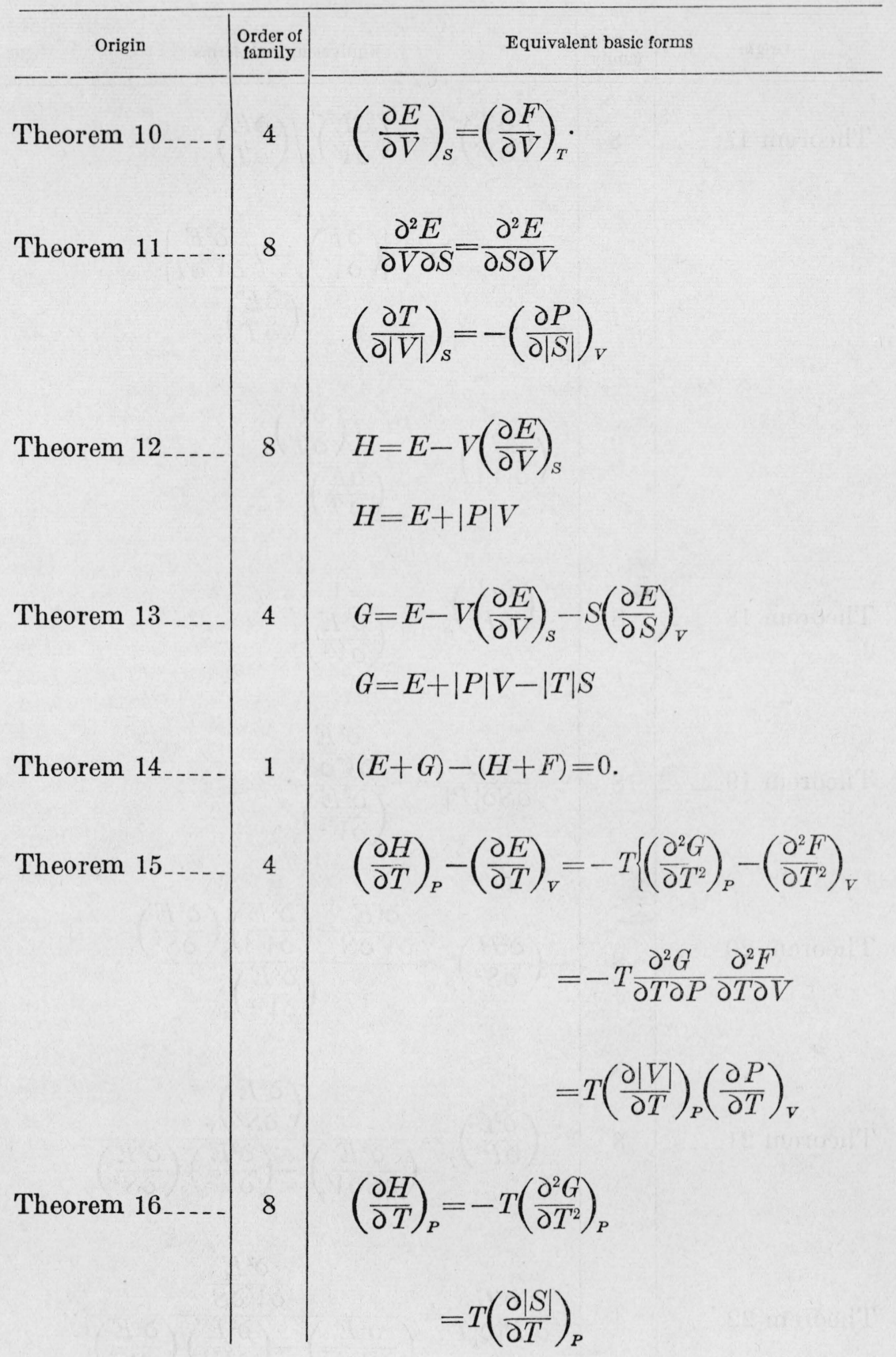


232 Journal of Research of the National Bureau of Standards

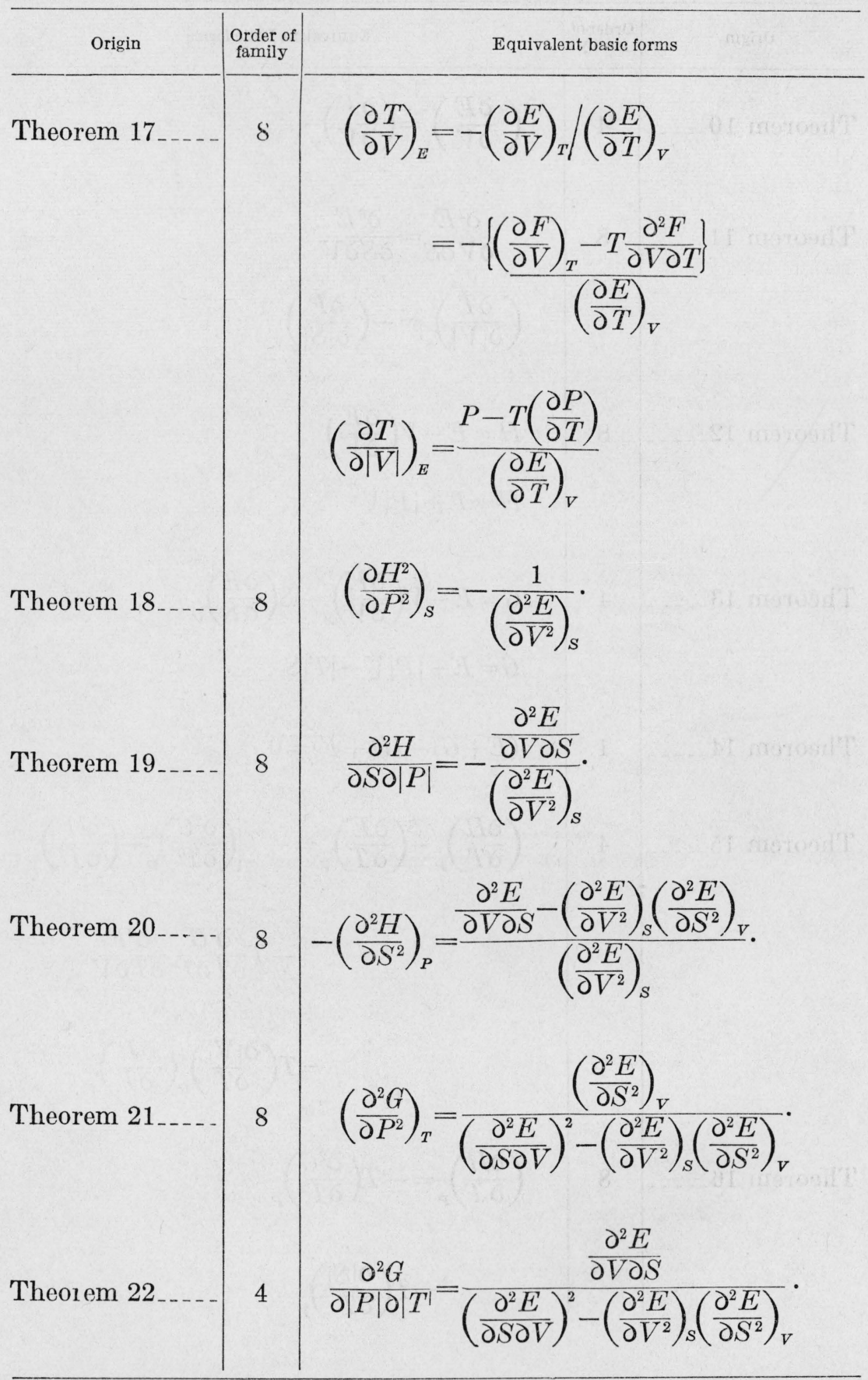


There are of course several equivalent expressions for a basic form involving second-order derivatives of the thermodynamic functions when expressed in terms of $V, S, T, P$. Since this paper is not concerned with providing a catalog of all such formulas they have been omitted. In fact, from the theoretical point of view, all formulas should remain implicitly contained in the general relations between the thermodynamic functions and their derivatives. Such a procedure would effect a considerable condensation of necessary formulas without entailing excessive effort in the deduction of a required relation.

Relations connecting the second-order derivatives of $V, S, T, P$, can be deduced from transformation formulas for the third-order derivatives of the characteristic functions. The relations are not complex, but there are many of them. In light of the results of this study, it is very probable that these equations can be grouped into families invariant under the substitution group $\mathscr{G}^{*}$, thereby eliminating an extensive catalog of formulas.

The writer is particularly indebted to A. C. Lunn, of the University of Chicago, for his inspiring lectures on group theory and theoretical physics and to F. O. Koenig, of Stanford University, for a criticism of an earlier derivation of the group $\mathbf{G}$.

\section{REFERENCES}

[1] F. O. Koenig, Jrn. Chem. Phys. 3, 29 (1935)

[2] C. Caratheodory, Math. Ann. 67, 355 (1909)

[3] A. R. Forsyth, Theory of Differential Equations, vol. I, part I, chap. IX (Cambridge Univ. Press, 1890).

[4] A. R. Forsyth, Theory of Differential Equations vol. V, part IV, chap. IX (Cambridge Univ. Press, 1906).

[5] S. Lie and E. Engle, Theorie der Transformationgruppen 2, 204, 209 (B. G. Teubner, Leipsig, 1890).

Washington, April 27, 1944. 
NATIONAL BUREAU OF STANDARDS, WASHINGTON, D. C.

Send me the Mathematical Tables marked $\times$ below. I enclose remittance to cover the cost.

\begin{tabular}{|c|c|c|c|c|}
\hline Mark X & Title of publication & $\begin{array}{l}\text { United States and } \\
\text { its possessions, } \\
\text { and countries ex- } \\
\text { tending franking } \\
\text { privilege }\end{array}$ & Other countries & Amount enclosed \\
\hline $\begin{array}{l} \\
\end{array}$ & 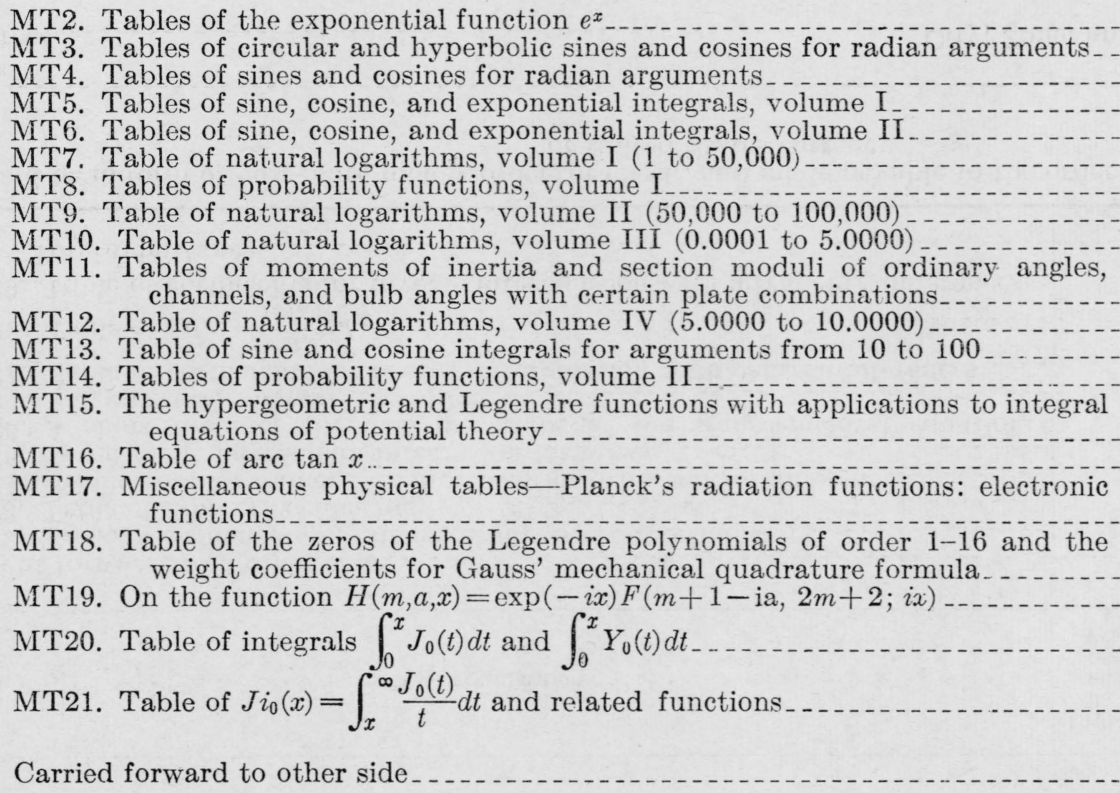 & $\begin{array}{r}\$ 2.00 \\
2.00 \\
2.00 \\
2.00 \\
2.00 \\
2.00 \\
2.00 \\
2.00 \\
2.00 \\
2.00 \\
2.00 \\
2.00 \\
2.00 \\
2.00 \\
2.00 \\
1.50 \\
\\
.25 \\
.25 \\
.25 \\
.25\end{array}$ & $\begin{array}{r}\$ 2.50 \\
2.50 \\
2.50 \\
2.50 \\
2.50 \\
2.50 \\
2.50 \\
2.50 \\
2.50 \\
2.50 \\
2.50 \\
2.50 \\
2.50 \\
2.50 \\
2.50 \\
1.75 \\
\\
.30 \\
.30 \\
.30 \\
\\
.30\end{array}$ & (1) \\
\hline
\end{tabular}




\begin{tabular}{|c|c|c|c|c|}
\hline Mark X & Title of publication & $\begin{array}{l}\text { United States and } \\
\text { its possessions, } \\
\text { and countries ex- } \\
\text { tending franking } \\
\text { privilege }\end{array}$ & Other countries & Amount enclosed \\
\hline (1, & $\begin{array}{l}\text { Brought forward from other side } \\
\text { MT22. Table of coefficients in numerical integration formulae } \\
\text { MT23. Table of Fourier coefficients } \\
\text { MT24. Coefficients for numerical differentiation with central differences } \\
\text { MT25. Seven-point Lagrangian integration formulas } \\
\text { MT26. A short table of the first five zeros of the transcendental equation, } \\
\text { MT27. Table of coefficients for inverse interpolation with central differences } J_{0}(x) Y_{0}(k x)-J_{0}(k x) Y_{0}(x)=0 \\
\text { MT28. Table of } f_{n}(x)=\frac{n !}{(x / 2)} J_{n}(x) \\
\text { MT29. Table of coefficients for inverse interpolation with advancing differences } \\
\text { Total remittance. }\end{array}$ & $\begin{array}{r}\$ 0.25 \\
.25 \\
.25 \\
.25 \\
.25 \\
.25 \\
.25 \\
.25\end{array}$ & $\begin{array}{r}30 \\
30 \\
30 \\
30 \\
30 \\
30 \\
.30 \\
30\end{array}$ & \begin{tabular}{|}
$\$ \ldots$ \\
\\
\end{tabular} \\
\hline
\end{tabular}

Remittance should be in form of post-office money order, or check, and made payable to the order of the "National Bureau of Standards" in United States currency

Send to

Number and Street.

City, Zone, and State 


\section{MATHEMATICAL TABLES}

Attention is invited to a series of publications prepared by the Project for the Computation of Mathematical Tables conducted by the Federal Works Agency, Work Projects Administration for the City of New York, under the sponsorship of the National Bureau of Standards. The tables which have been made available through the National Bureau of Standards are listed below. A list of other WPA tables obtainable elsewhere will be sent by the Bureau on request.

There is included in this list a publication on the hypergeometric and Legendre functions (MT15), prepared by the Bureau.

MT1. Table of the First Ten Powers of the Integers From 1 to 1000: (1938) VIII+80 pages; heavy paper cover. Out of print.

MT2. Tables of the Exponential Function $e^{x}$ : The ranges and intervals of the argument and the number of decimal places in the entries are given below:

$\begin{array}{rcc}\text { Range of } x & \text { Interval of } x & \text { Decimals given } \\ -2.5000 \text { to } 1.0000 & 0.0001 & 18 \\ 1.0000 \text { to } 2.5000 & .0001 & 15 \\ 2.500 \text { to } 5.000 & .001 & 15 \\ 5.00 \text { to } 10.00 & .01 & 12\end{array}$

(1939) XV+535 pages; bound in buckram, $\$ 2.00$.

MT3. Tables of Circular and Hyperbolic Sines and Cosines for Radian Arguments: Contains 9 decimal place values of $\sin x, \cos x, \sinh x$, and $\cosh x$ for $x$ (in radians) ranging from 0 to 2 at intervals of 0.0001 . (1939) XVII+ 405 pages; bound in buckram, $\$ 2.00$.

MT4. Tables of Sines and Cosines for Radian Arguments: Contains 8 decimal place values of sines and cosines for radian arguments ranging from 0 to 25 at intervals of 0.001 . (1940) XXIX +275 pages; bound in buckram, $\$ 2.00$.

MT5. Tables of Sine, Cosine, and Exponential Integrals, Volumb I: Values of these functions to 9 places of decimals from 0 to 2 at intervals of 0.0001 . (1940) XXVI+ 444 pages; bound in buckram, $\$ 2.00$.

MT6. Tables of Sine, Cosine, and Exponential Integrals, Volume II: Values of these functions to 9,10 , or 11 significant figures from 0 to 10 at intervals of 0.001 with auxiliary tables. (1940) XXXVII+ 225 pages; bound in buckram, $\$ 2.00$.

MT7. Table of Natural Logarithms, Volume I: Logarithms of the integers from 1 to 50,000 to 16 places of decimals. (1941) XVIII+501 pages; bound in buckram, $\$ 2.00$.

MT8. Tables of Probability Functions, Volume I: Values of these functions to 15 places of decimals from 0 to 1 at intervals of 0.0001 and from 1 to 5.6 at intervals of 0.001 . (1941) XXVIII+302 pages; bound in buckram, $\$ 2.00$.

MT9. Table of Natural Logarithms, Volume II: Logarithms of the integers from 50,000 to 100,000 to 16 places of decimals. (1941) XVIII + 501 pages; bound in buckram, $\$ 2.00$.

MT10. Table of Natural Logarithms, Volume III: Logarithms of the decimal numbers from 0.0001 to 5.0000 , to 16 places of decimals. (1941) XVIII +501 pages; bound in buckram, $\$ 2.00$.

MT11. Tables of the Moments of Inertia and Section Moduli of Ordinary Angles, Channels, and Bulb Angles with Certain Plate Combinations: (1941) XIII+197 pages; bound in green cloth. $\$ 2.00$.

MT12. Table of Natural Logarithms, Volume IV: Logarithms of the decimal numbers from 5.0000 to 10.0000 , to 16 places of decimals. (1941) XXII + 506 pages; bound in buckram, $\$ 2.00$.

MT13. Table of Sine and Cosine Integrals for Arguments from 10 to 100: (1942) XXXII+185 pages; bound in buckram, $\$ 2.00$.

MT14. Tables of Probability Functions, Volume II: Values of these functions to 15 places of decimals from 0 to 1 at intervals of 0.0001 and from 1 to 7.8 at intervals of 0.001 . (1942) XXI+344 pages; bound in buckram, $\$ 2.00$.

MT15. The Hypergeometric and Legendre Functions With Applications to Integral Equa. tions of Potential Theory. By Chester Snow, National Bureau of Standards. Reproduced from original handwritten manuscript. (1942) VII +319 pages, bound in heavy paper cover. $\$ 2.00$.

MT16. TABLe of ARC TAN $x$ : Table of inverse tangents for positive values of the angle in radians. Second central differences are included for all entries. $x=\llbracket 0(.001) 7(.01) 50(.1) 300(1) 2,000(10)$ 10,$000 ; 12 D \rrbracket(1942) \mathrm{XXV}+169$ pages; bound in buckram, $\$ 2.00$. 
[Continued from p. 3 of cover.]

MT17. Miscellanbous Physical Tables: Planck's Radiation Functions (Originally published in the Journal of the Optical Society of America, February 1940); and Electronic Funcrions. (1941) VII+58 pages; bound in buckram, $\$ 1.50$.

MT18. Table of the Zeros of the Legendre Polynomials of Order 1-16 and the Weight Coepricients for Gauss' Mechanical Quadrature Formula. (Reprinted from Bul. Amer. Mathematical Society, October 1942.) 5 pages, with cover, 25 cents.

MT19. On the Function $H(m, a, x)=\operatorname{Exp}(-i x) F(m+1-i a, 2 m+2 ; i x)$; with table of the confluent hypergeometric function and its first derivative. (Reprinted from J. Math. Phys., December 1942.) 20 pages, with cover, 25 cents.

MT20. Table of Integrals $\int_{0}^{x} J_{0}(t) d t$ and $\int_{0}^{x} \mathrm{Y}_{0}(t) d t$. (Reprinted from J. Math. Phys., May 1943.) 12 pages, with cover, 25 cents.

MT21. Table of $J i_{0}(x)=\int_{x}^{\infty} \frac{J_{0}(t)}{t} d t$ and Related Functions. (Reprinted from J. Math. Phys., June 1943.) 7 pages, with cover, 25 cents.

MT22. Table of Cobpricients in Numerical Intrgration Formulae. (Reprinted from J. Math. Phys., June 1943.) 2 pages, with cover, 25 cents.

MT23. Table op Fourier Coepricients. (Reprinted from J. Math. Phys. Sept. 1943.) 11 pages, with cover, 25 cents.

MT24. Coepricients por Numerical Differentiation With Central Difrerences. (Reprinted from J. Math. Phys., Sept. 1943.) 21 pages, with cover, 25 cents.

MT25. Seven-Point Lagrangian Intrgration Formulas. (Reprinted from J. Math. Phys., Dec. 1943.) 4 pages, with cover, 25 cents.

MT26. A Short Table of the First Five Zeros of the Transcendental Enuation $J_{0}(x) \Upsilon_{0}(k x)-J_{0}(k x) \Upsilon_{0}(x)=0$. (Reprinted from J. Math. Phys., Dec. 1943.) 2 pages, with cover 25 cents.

MT27. Table of Corpricients for Inverse Interpolation with Central Difperences. (Re. printed from J. Math. Phys., Dec. 1943.) 15 pages, with cover. 25 cents.

MT28. TABLr or $f_{n}(x)=\frac{n !}{(x / 2)^{n}} J_{n}(x)$. (Reprinted from J. Math. Phys., Feb. 1944.) 16 pages, with cover, 25 cents.

MT29: Table of Corppicients for Inverse Interpolation with Advancing Diprerences. (Reprinted from J. Math. Phys., May 1944.) 28 pages, with cover, 25 cents.

Payment is required in advance. Make remittance payable to the "National Bureau of Standards" and send with order, using the blank form facing page 3 of the cover.

A mailing list is maintained for those who desire to receive announcements regarding new tables as they become available. 